\title{
GCU
}

Glasgow Caledonian

University

University for the Common Good

\section{Effectiveness of needle/syringe programmes and opiate substitution therapy in preventing HCV transmission among people who inject drugs}

Platt, L.; Reed, J.; Minozzi, S.; Vickerman, P.; Hagan, H.; French, C.; Jordan, A.; Degenhardt, L.; Hope, V.; Hutchinson, S.; Maher, L.; Palmateer, N.; Taylor, A.; Hickman, M.

Published in:

Cochrane Database of Systematic Reviews

DOI:

10.1002/14651858.CD012021

Publication date:

2016

Document Version

Publisher's PDF, also known as Version of record

Link to publication in ResearchOnline

Citation for published version (Harvard):

Platt, L, Reed, J, Minozzi, S, Vickerman, P, Hagan, H, French, C, Jordan, A, Degenhardt, L, Hope, V, Hutchinson, S, Maher, L, Palmateer, N, Taylor, A \& Hickman, M 2016, 'Effectiveness of needle/syringe programmes and opiate substitution therapy in preventing HCV transmission among people who inject drugs', Cochrane Database of Systematic Reviews , vol. 2016, no. 1, CD012021.

https://doi.org/10.1002/14651858.CD012021

\section{General rights}

Copyright and moral rights for the publications made accessible in the public portal are retained by the authors and/or other copyright owners and it is a condition of accessing publications that users recognise and abide by the legal requirements associated with these rights.

Take down policy

If you believe that this document breaches copyright please view our takedown policy at https://edshare.gcu.ac.uk/id/eprint/5179 for details of how to contact us. 


\section{Cochrane}

Cochrane Database of Systematic Reviews

\section{Effectiveness of needle/syringe programmes and opiate substitution therapy in preventing HCV transmission among people who inject drugs (Protocol)}

Platt L, Reed J, Minozzi S, Vickerman P, Hagan H, French C, Jordan A, Degenhardt L, Hope V, Hutchinson S, Maher L, Palmateer N, Taylor A, Hickman M

Platt L, Reed J, Minozzi S, Vickerman P, Hagan H, French C, Jordan A, Degenhardt L, Hope V, Hutchinson S, Maher L, Palmateer N, Taylor A, Hickman M.

Effectiveness of needle/syringe programmes and opiate substitution therapy in preventing HCV transmission among people who inject drugs. Cochrane Database of Systematic Reviews 2016, Issue 1. Art. No.: CD012021.

DOI: 10.1002/14651858.CD012021.

www.cochranelibrary.com

Effectiveness of needle/syringe programmes and opiate substitution therapy in preventing HCV transmission among people who inject drugs (Protocol)

Copyright @ 2016 The Cochrane Collaboration. Published by John Wiley \& Sons, Ltd. 
TABLE OF CONTENTS

HEADER . . . . . . . . . . . . . . . . . . . . . . . . . . . . . . . . . . . 1

ABSTRACT . . . . . . . . . . . . . . . . . . . . . . . . . . . . . . . . . . . . . . . . . . . . .

BACKGROUND . . . . . . . . . . . . . . . . . . . . . . . . . . . . . . . . . . . . . 2

OBJECTIVES . . . . . . . . . . . . . . . . . . . . . . . . . . . . . . . . . . . . . . .

METHODS . . . . . . . . . . . . . . . . . . . . . . . . . . . . . . . . . . . . . .

REFERENCES . . . . . . . . . . . . . . . . . . . . . . . . . . . . . . . . . . . . . 6

APPENDICES . . . . . . . . . . . . . . . . . . . . . . . . . . . . . . . . . . . . . . 8

WHAT'S NEW . . . . . . . . . . . . . . . . . . . . . . . . . . . . . . . . . . 12

CONTRIBUTIONS OF AUTHORS . . . . . . . . . . . . . . . . . . . . . . . . . . . . . . . . . . .

DECLARATIONS OF INTEREST . . . . . . . . . . . . . . . . . . . . . . . . . . . . . . . . 12

SOURCES OF SUPPORT . . . . . . . . . . . . . . . . . . . . . . . . . . . . . . . . . . . . . . . .

Effectiveness of needle/syringe programmes and opiate substitution therapy in preventing HCV transmission among people who inject drugs (Protocol)

Copyright $\odot 2016$ The Cochrane Collaboration. Published by John Wiley \& Sons, Ltd. 


\section{[Intervention Protocol] \\ Effectiveness of needle/syringe programmes and opiate substitution therapy in preventing HCV transmission among people who inject drugs}

Lucy Platt ${ }^{1}$, Jennifer Reed ${ }^{2}$, Silvia Minozzi ${ }^{3}$, Peter Vickerman ${ }^{4}$, Holly Hagan $^{2}$, Clare French $^{4}$, Ashly Jordan ${ }^{2}$, Louisa Degenhardt ${ }^{5}$, Vivian Hope ${ }^{6}$, Sharon Hutchinson ${ }^{7}$, Lisa Maher ${ }^{8}$, Norah Palmateer ${ }^{7}$, Avril Taylor ${ }^{9}$, Matthew Hickman ${ }^{4}$

${ }^{1}$ Centre for Research on Drugs and Health Behaviour, Department of Social and Environmental Health Research, London School of Hygiene and Tropical Medicine, London, UK. ${ }^{2}$ New York University College of Nursing, New York, NY, USA. ${ }^{3}$ Department of Epidemiology, Lazio Regional Health Service, Rome, Italy. ${ }^{4}$ School of Social and Community Medicine, University of Bristol, Bristol, UK. ${ }^{5}$ National Drug and Alcohol Research Centre, UNSW, Randwick, Australia. ${ }^{6}$ Public Health England, London, UK. ${ }^{7}$ Health Protection Scotland, Glasgow, UK. ${ }^{8}$ Kirby Institute, University of New South Wales, Sydney, Australia. ${ }^{9}$ University of West of Scotland, Paisley, UK

Contact address: Lucy Platt, Centre for Research on Drugs and Health Behaviour, Department of Social and Environmental Health Research, London School of Hygiene and Tropical Medicine, 15 - 17 Tavistock Place, London, WC1H 9SH, UK. lucy.platt@lshtm.ac.uk.

Editorial group: Cochrane Drugs and Alcohol Group.

Publication status and date: Edited (no change to conclusions), published in Issue 1, 2016.

Citation: Platt L, Reed J, Minozzi S, Vickerman P, Hagan H, French C, Jordan A, Degenhardt L, Hope V, Hutchinson S, Maher L, Palmateer N, Taylor A, Hickman M. Effectiveness of needle/syringe programmes and opiate substitution therapy in preventing HCV transmission among people who inject drugs. Cochrane Database of Systematic Reviews 2016, Issue 1. Art. No.: CD012021. DOI: 10.1002/14651858.CD012021.

Copyright (C) 2016 The Cochrane Collaboration. Published by John Wiley \& Sons, Ltd.

\section{A B S T R A C T}

This is the protocol for a review and there is no abstract. The objectives are as follows:

1. To assess the impact of needle/syringe programmes with and without opiate substitution therapy (OST) on the incidence of $\mathrm{HCV}$ infection among people who inject drugs (PWID).

2. To assess the effect of OST alone on the incidence of HCV infection among PWID.

Research questions

1. How effective are needle/syringe programmes (NSP) with and without the use of OST for reducing HCV incidence among PWID?

2. How effective is OST alone for reducing HCV incidence among PWID?

3. How does the effect of NSP and OST vary according to duration of treatment (i.e. for NSPs weekly attendance versus monthly)?

4. How does the effect of NSP vary according to the type of service (fixed site versus mobile; high coverage versus low coverage)?

5. How does the effect of OST vary according to the dosage of OST, type of substitution used and adherence to treatment?

Effectiveness of needle/syringe programmes and opiate substitution therapy in preventing HCV transmission among people who inject drugs (Protocol)

Copyright $(2016$ The Cochrane Collaboration. Published by John Wiley \& Sons, Ltd. 


\section{B A C K G R O U N D}

\section{Description of the condition}

The number of people with hepatitis $\mathrm{C}$ virus ( $\mathrm{HCV}$ ) continues to increase globally, with an estimated 114.9 (91.9 to 148.7) million people infected with antibodies to HCV (Gower 2014), 3 to 4 million people newly infected each year and 350,000 deaths occurring annually (Mohd Hanafiah 2013; Perz 2006). There were an estimated 35 million people living with human immunodeficiency virus (HIV) in 2014. Emerging evidence suggests that HIV transmission has declined since 2001 and more people are being treated (UNAIDS 2014). Co-infection of HCV among people living with HIV is a major global public health concern, with an estimated 4 million people co-infected (Platt 2015).

Evidence shows that injecting with needles/syringes that have been used by someone else is the main risk factor for infection with HIV and HCV for people who inject drugs (PWID). Additional risks for $\mathrm{HCV}$ include sharing drug preparation containers, filters and rinse water, and back loading (a method of sharing drugs by injecting them from one syringe into the back of another opened syringe) (Pouget 2012; Strathdee 2010).

\section{Description of the intervention}

Needle and syringe programmes (NSPs) are often a first point of contact for PWID. They provide immediate support to minimise drug and sexual risk related harms, including the provision of clean needles/syringes and condoms to prevent the transmission of blood borne viruses and bacterial infections, and other adverse health outcomes. By maximising the amount of clean injecting equipment in circulation, it is possible to minimise the time that infected equipment remains in use and the proportion of unsafe injections (Kaplan 1992). NSPs operate through a range of modalities including via fixed-sites, outreach, peer PWID networks, vending machines, and pharmacies. Engaging in behaviours that are socially stigmatised and illegal, PWID have high rates of unemployment and homelessness, and have frequently been in prison. NSPs also provide access to longer-term support by engaging with users to make onward referrals to other much-needed medical, drug treatment or social support services.

Drug treatment for opiate addiction and dependence also encompasses a range of strategies to manage injecting drug use, such as pharmacological maintenance or detoxification treatment, pharmacotherapy combined with psychosocial approaches and residential rehabilitation. The most commonly prescribed forms of opioid substitution therapy (OST) are opiate agonist treatments: methadone maintenance therapy and buprenorphine maintenance treatment. OST is prescribed to dependent users to diminish the use and effects of illicitly-acquired opiates. It is usually taken orally and therefore reduces the frequency of injection and unsafe injecting practices (Tilson 2007). Evidence suggests that OST is most effective when it is continuous and provided at adequate doses (Amato 2013; Faggiano 2003).

International evidence supports the use of combination interventions to prevent and treat HIV among PWID with the provision of needles/syringe programmes, antiretroviral treatment and opiate substitution therapy as the cornerstone interventions (Degenhardt 2010). There is good evidence that NSP and OST in combination reduce injecting risk behaviours, and evidence to show an impact on HIV incidence; however, evidence of their impact on $\mathrm{HCV}$ incidence among PWID in combination or alone is limited ( Gibson 2001; Gowing 2011; Jones 2011; Palmateer 2010; Van Den Berg 2007).

\section{How the intervention might work}

Two recent systematic reviews of 12 observational studies estimated a moderate effect of NSPs on reducing HIV transmission by $48 \%$ (95\% confidence interval (CI) $3 \%$ to $72 \%$ ) and strong evidence for OST reducing HIV transmission by $54 \%$ (95\% CI $33 \%$ to $68 \%$ ) (Aspinall 2014; MacArthur 2012). Previous reviews (Gibson 2001; Tilson 2007; Wodak 2004) have synthesised evidence for the use of NSPs but focused primarily on HIV as the main outcome and as a consequence failed to include all the available evidence on HCV (Palmateer 2010).

A recent analysis of pooled data $(\mathrm{N}=919)$ identified through a systematic review examined the effect of NSP coverage on HCV incidence, defining coverage in terms of the proportion of injections with a sterile syringe. This analysis suggested that high coverage of NSP (" $100 \%$ NSP”, i.e. obtaining $\geq 1$ sterile syringe per injection) or OST (defined as receiving OST or not, either currently or within the last 6 months) can each reduce HCV infection risk by $50 \%$; and in combination by $80 \%$ (Turner 2011 ). However, due to a small number of incident $\mathrm{HCV}$ cases $(\mathrm{N}=40)$, the efficacy estimate for $100 \%$ NSP among those not on OST was borderline significant (95\% CI 0.22 to 1.12 ), and there was insufficient power to evaluate whether a dose response relationship exists. Another systematic review examined evidence drawn from observational studies on a range of risk reduction interventions including behavioural interventions, NSP, and OST on HCV seroconversion (Hagan 2011). This study measured the effect of NSP use, defined inconsistently by available studies as any attendance at a NSP or attendance at one point in time, and showed increased risk of seroconversion. Limitations of the available studies that comprised this review included substantial heterogeneity, and lack of clarity and consistency in the measurement of NSP use across studies. That evidence was drawn only from North America limits the generalisability of findings to other geographic settings. A previous review on the effect of OST use on HIV transmission detected many more studies than earlier Cochrane reviews 
(MacArthur 2012). We also expect that not all evidence on the effect of NSP on HCV transmission has been identified. Extending previous reviews would strengthen the evidence base, as well as provide a more refined measure of NSP coverage that accounts for frequency and degree to which NSPs meet individuals' requirements for sterile drug injection equipment.

\section{Why it is important to do this review}

Evidence of the effect of NSP with and without OST on HCV incidence is inconclusive (Committee on the Prevention of HIV infection 2007; Mathers 2012). Previous reviews have failed to define the frequency of use of the intervention and the quantity of needles/syringes received by injectors (Hagan 2011), and a previous pooled analysis had an insufficient sample size to accurately measure the effect (Turner 2011). This review is needed in order to estimate the effect of NSPs using a consistent definition of coverage, and examining impact with and without OST on HCV incidence, in order to inform harm reduction policies to reduce the burden of HCV.

\section{O B J E C T IVES}

1. To assess the impact of needle/syringe programmes with and without opiate substitution therapy (OST) on the incidence of HCV infection among people who inject drugs (PWID).

2. To assess the effect of OST alone on the incidence of HCV infection among PWID.

\section{Research questions}

1. How effective are needle/syringe programmes (NSP) with and without the use of OST for reducing HCV incidence among PWID?

2. How effective is OST alone for reducing HCV incidence among PWID?

3. How does the effect of NSP and OST vary according to duration of treatment (i.e. for NSPs weekly attendance versus monthly)?

4. How does the effect of NSP vary according to the type of service (fixed site versus mobile; high coverage versus low coverage)?

5. How does the effect of OST vary according to the dosage of OST, type of substitution used and adherence to treatment?

\section{Criteria for considering studies for this review}

\section{Types of studies}

We will include randomised controlled trials (RCTs), prospective and retrospective cohort studies and case-control studies. Prospective studies that examine HCV incidence in PWID, which may have collected data regarding NSPs and OST but which have not reported the data in the published study, or have reported data as part of an adjusted analysis, will also be included. For these studies, we will seek unpublished data relating to the impact of NSP/OST on HCV transmission via contact with study authors. Studies will only be included if these data are provided.

Cross-sectional surveys will be used if they include a measure of recent infection through positive ribonucleic acid (RNA) results on anti-body negative samples. We will exclude cross-sectional studies (including serial cross-sectional studies) reporting $\mathrm{HCV}$ prevalence. Methods to be used in this systematic review in relation to the search strategies and approaches to data synthesis follow methods applied in a similar review to assess the impact of OST on HIV incidence (MacArthur 2012).

\section{Types of participants}

People who inject drugs (opiate and or stimulants). Studies that include participants undergoing opportunistic HCV testing (outside of the study setting) will be excluded, as will those relating to people who inject drugs in the prison setting, since treatment provision in this setting will differ significantly to community and healthcare settings.

\section{Types of interventions}

\section{Experimental interventions}

- NSP and OST

- NS alone

- OST alone

Studies may be based in a drug treatment facility or in the wider community, at a fixed site or mobile unit.

- Exposure to NSP will be defined as the proportion of injections that are covered by a clean needle/syringe. Where it is not possible to estimate this proportion we will define exposure accounting for frequency of injection and the degree to which the NSP meets the individual's requirement for needles/syringes.

- Exposure to OST will be defined as the continuous or interrupted treatment, current, recent (past 6 months or duration of HCV observation period) or any past treatment with methadone or buprenorphine.

\section{METHODS}

Effectiveness of needle/syringe programmes and opiate substitution therapy in preventing HCV transmission among people who inject 


\section{Control intervention: \\ - no intervention; \\ - OST alone.}

\section{Types of comparisons}

1. NSP with OST versus NSP alone;

2. NSP with OST versus OST alone;

3. NSP with OST versus no intervention;

4. NSP alone versus no intervention;

5. OST alone versus no intervention.

\section{Types of outcome measures}

\section{Primary outcomes}

Incidence of $\mathrm{HCV}$ infection in PWID as measured via repeat testing such as detection of HCV RNA positive among HCV antibody negative results or antibody avidity.

Studies will be included if they report a minimum of two HCV seroconversions (HCV antibody negative to HCV antibody positive) among the study participants from tests conducted at different time points. We will exclude studies relying on self-reported data for the outcome.

\section{Search methods for identification of studies}

Papers will be identified in four ways. Firstly we will conduct two primary searches of the literature based on key search terms identified by the review of reviews and recent review of the effect of OST and NSP on the risk of HIV and HCV among PWID (MacArthur 2012; Palmateer 2010). The purpose of the two searches are to: a) to identify studies that measure the impact of NSP/OST on HCV incidence (see Appendix 1) and b) to identify longitudinal studies that measure HCV incidence and report the impact of NSP/OST as part of an adjusted analysis (see Appendix 2). The search strategy will be reviewed by the Cochrane Drugs and Alcohol Group Trials Search Co-ordinator, who will also conduct the search.

\section{Electronic searches}

We will search for relevant studies in the following sources:

- the Cochrane Drugs and Alcohol Group's Specialised

Register of Trials;

- the Cochrane Central Register of Controlled Trials

(CENTRAL, most recent issue);

- the Cochrane Database of Systematic Reviews (CDSR)

(Cochrane Library, most recent issue);

- the Database of Abstracts of Reviews of Effects (DARE)

(Cochrane Library, most recent issue);

- MEDLINE (Ovid) (1966 to present);
- EMBASE (EMBASE.com) (1974 to present);

- Global Health (Ovid) (1974 to present);

- CINAHL (EBSCOhost) (1982 to present);

- Web of Science (1991-to present);

- PsycINFO (Ovid) (1985 to present).

We will search for ongoing clinical trials and unpublished trials via searches of the following websites:

- ClinicalTrials.gov (www.clinicaltrials.gov);

- World Health Organisation (WHO) International Clinical Trials Registry Platform (ICTRP) (apps.who.int/trialsearch/).

\section{Searching other resources}

We will search the publications of key international agencies including the European Monitoring Centre on Drugs and Drug Addiction, European Centre for Disease Control, the National Institute on Drug Abuse, the US Institute of Medicine, the United Nations Office on Drugs and Crime Prevention and the World Health Organisation. We will handsearch the reference lists of relevant articles to identify additional relevant studies, and contact experts in the field to identify ongoing research. We will also search conference abstracts including the International Harm Reduction Conference, International HIV/AIDS Society and the European Association for the Study of the Liver conference. Finally we will contact principal investigators and authors of prospective studies that examine HCV incidence in PWID to collate data regarding NSPs and OST but which have not reported the data in the published study,

There will be no language or date restrictions, and we will include peer reviewed and non-peer reviewed papers.

\section{Data collection and analysis}

\section{Selection of studies}

Papers will be screened according to the title and abstract (where available) by two review authors independently using the inclusion and exclusion criteria outlined above. Full text papers will be obtained and screened by two review authors independently. Disagreement will be resolved by discussion or, where disagreements persist, by a third author, to enable a consensus to be reached. Where there are multiple publications from the same study, or the same city or region, all published papers will be selected and data extracted from the study with the greatest number of outcome events, i.e. HCV seroconversions.

\section{Data extraction and management}

Data will be extracted by one author using a data extraction form in Excel. For 10\% of included studies, data will be double extracted 
by a second author in order to assess the accuracy of data extraction. Prior to extraction, the data extraction form will be piloted by two review authors to assess its suitability for the capture of study data and assessment of quality. Data to be extracted include:

- Lead author, review title or unique identifier and date

- Eligibility for inclusion

- Reasons for exclusion

- Study aim(s)

- Study design (included sampling methods, participant and attrition rate)

- Study location

- Study setting

- Proportion of participants who are opiate injectors

- Proportion of participants who are stimulant injectors

- Definition of exposure (recency of injecting)

- Intervention (NSP provision; number of needles distributed; frequency of injection; methadone maintenance therapy or buprenorphine maintenance treatment; delivery (e.g. continuous versus interrupted treatment); duration; dose)

- Additional interventions or incentives provided alongside NSP/OST

- Participants (number in each intervention group; age, gender and ethnicity)

- Duration of follow up in each treatment arm

- Outcome measure (HCV seroconversion) overall and by NSP and OST exposure

- Unadjusted and adjusted effect size (incidence rate ratio (IRR); odds ratio (OR); hazard ratio (HR) and precision (e.g. 95\% confidence interval (CI))

- Confounding factors used to adjust effect estimates including high-risk behaviours (injecting risk behaviours, frequency of injection, homelessness, experience of prison, duration of injection, or age, poly drug use)

- Background prevalence of HCV in the population

- Any other comments

\section{Assessment of risk of bias in included studies}

The 'Risk of bias' assessment for RCTs will be performed using the criteria recommended in the Cochrane Handbook (Higgins 2011). The recommended approach is a two-part tool, addressing seven specific domains, namely sequence generation and allocation concealment (selection bias), blinding of participants and providers (performance bias), blinding of outcome assessor (detection bias), incomplete outcome data (attrition bias), selective outcome reporting (reporting bias) and other source of bias. The first part of the tool involves describing what was reported to have happened in the study. The second part of the tool involves assigning a judgement relating to the risk of bias for that entry, in terms of low, high or unclear risk. To make these judgments we will use the criteria indicated by the Handbook, adapted to the addiction field. See Appendix 3 for details. Assessment of risk of bias for unpublished estimates will be made by referring to the study methods in the corresponding published paper.

We will assess the risk of bias in non-randomised studies using a pilot version of a tool in development by Methods Groups of the Cochrane Collaboration (Sterne 2013). This will be undertaken as part of the formal piloting of the tool, in collaboration with its developers. The tool is an extension of the existing tool for assessing risk of bias in randomised trials (Higgins 2011).

It is composed of six domains, two concerning the pre-intervention phase:

1. bias due to baseline confounding

2. bias in selection of participants into the study and four the post-intervention phase:

1. bias due to departures from intended interventions (performance bias)

2. bias due to missing data (attrition bias)

3. bias in measurement of outcomes or Interventions (detection bias)

4. bias in selection of the reported results (outcome reporting bias).

If the pilot version of the tool changes during the course of the review, we may update our 'Risk of bias' assessments accordingly.

\section{Grading of evidence}

We will assess the overall quality of the evidence for the primary outcome using the GRADE system. The Grading of Recommendation, Assessment, Development and Evaluation (GRADE) Working Group developed a system for grading the quality of evidence (GRADE 2004; Guyatt 2008; Guyatt 2011; Schünemann 2006) which takes into account issues not only related to internal validity but also to external validity, such as directness of results. The 'Summary of findings' tables present the main findings of a review in a transparent and simple tabular format. In particular, they provide key information concerning the quality of evidence, the magnitude of effect of the interventions examined and the sum of available data on the main outcomes.

The GRADE system uses the following criteria for assigning grades of evidence:

High: further research is very unlikely to change our confidence in the estimate of effect. Moderate: further research is likely to have an important impact on our confidence in the estimate of effect and may change the estimate. Low: further research is very likely to have an important impact on our confidence in the estimate of effect and is likely to change the estimate. Very low: any estimate of effect is very uncertain.

Grading is decreased for the following reasons: Serious (-1) or very serious (-2) limitation to study quality. Important inconsistency ($1)$. Some (-1) or major (-2) uncertainty about directness. Imprecise or sparse data $(-1)$. High probability of reporting bias $(-1)$.

Grading is increased for the following reasons: Strong evidence of association - significant relative risk of $>2(<0.5)$ based on 
consistent evidence from two or more observational studies, with no plausible confounders $(+1)$. Very strong evidence of association - significant relative risk of $>5(<0.2)$ based on direct evidence with no major threats to validity $(+2)$. Evidence of a dose response gradient $(+1)$. All plausible confounders would have reduced the effect $(+1)$.

\section{Measures of treatment effect}

Dichotomous outcomes will be analysed by calculating the relative risk (RR) for each study, with the uncertainty in each result expressed with $95 \%$ confidence intervals (CI). Continuous outcomes will be analysed by calculating the mean difference (MD) or the standardised mean difference (SMD) with $95 \%$ CI.

\section{Dealing with missing data}

Study authors will be contacted if studies provide data regarding use of NSP or the impact of drug treatment on HCV transmission, but where insufficient detail is provided regarding the precise form of treatment provided. We will also contact study authors if $\mathrm{HCV}$ incidence data are reported but no data regarding drug treatment or NSP are provided. If missing data cannot be obtained, studies will be excluded from analysis.

\section{Assessment of heterogeneity}

Heterogeneity will be examined via inspection of the forest plot and by a $\mathrm{Chi}^{2}$ test to demonstrate whether the observed differences in results are compatible with chance alone. The $\mathrm{I}^{2}$ statistic will be calculated to examine the percentage of variability that is due to heterogeneity rather than to sampling error. Heterogeneity will be explored through sensitivity and subgroup analysis

\section{Assessment of reporting biases}

Funnel plots will be used to plot the study effect size against sample size to assess publication bias.

\section{Data synthesis}

Since there may be significant heterogeneity between studies, a description and summary of the findings of included studies will be included in the review. Where studies are sufficiently similar, we will carry out meta-analysis. The appropriate method of metaanalysis will depend on the nature of the outcome data (dichotomous, ordinal, continuous, time-to-event etc.)

A random-effects model will be used because some degree of heterogeneity is expected. We will conduct subgroup analysis by study design if there is a great variation in study design.

\section{Subgroup analysis and investigation of heterogeneity}

Where sufficient data are available, we will perform subgroup analyses and meta-regression models to compare outcomes by:

- Age, gender and/or ethnicity

- Frequency of injecting and main drug injected

- Dose, duration, and/or delivery of NSP/ OST (i.e.

continuous or interrupted treatment)

- Type of OST provided (i.e. methadone maintenance therapy or buprenorphine maintenance treatment)

- Type of NSP (fixed site or mobile)

\section{Sensitivity analysis}

We will assess whether meta-analyses are subject to small study bias by carrying out fixed-effect and random-effects meta-analyses. We will exclude studies that are at critical risk of bias. We will also use sensitivity analysis to determine to what extent the overall intervention effect is changed by the inclusion of: studies at severe or unclear risk of bias; studies that did not adjust for confounders; studies that measured incidence rate ratio; and studies that assessed the impact of intervention at baseline only.

\section{R E F E R E N C E S}

\section{Additional references}

Amato 2013

Amato L, Davoli M, Minozzi S, Ferroni E, Ali R, Ferri M. Methadone at tapered doses for the management of opioid withdrawal. Cochrane Database of Systematic Reviews 2013, Issue 2. [DOI: 10.1002/14651858.CD003409.pub4]

Aspinall 2014

Aspinall EJ, Nambiar D, Goldberg DJ, Hickman M, Weir A, Van Velzen E, et al. Are needle and syringe programmes associated with a reduction in HIV transmission among people who inject drugs: a systematic review and meta- analysis. International Journal of Epidemiology 2014;43(1): 235-48.

Committee on the Prevention of HIV infection 2007 Committee on the Prevention of HIV infection among Injecting Drug Users in High-Risk Countries. Preventing HIV infection among injecting drug users in high-risk countries: an assessment of the evidence. Washington, DC,: Institute of Medicine, 2007.

Degenhardt 2010

Degenhardt L, Mathers B, Vickerman P, Rhodes T, Latkin C, Hickman M. Prevention of HIV infection for people who

Effectiveness of needle/syringe programmes and opiate substitution therapy in preventing HCV transmission among people who inject 
inject drugs: why individual, structural, and combination approaches are needed. Lancet 2010;376(9737):285-301.

Faggiano 2003

Faggiano F, Vigna-Taglianti F, Versino E, Lemma P. Methadone maintenance at different dosages for opioid dependence. Cochrane Database of Systematic Reviews 2003, Issue 3. [DOI: 10.1002/14651858.CD002208]

\section{Gibson 2001}

Gibson DR, Flynn NM, Perales D. Effectiveness of syringe exchange programs in reducing HIV risk behavior and HIV seroconversion among injecting drug users. AIDS 2001;15 (11):1329-41.

\section{Gower 2014}

Gower E, Estes C, Blach S, Razavi-Shearer K, Razavi H. Global epidemiology and genotype distribution of the hepatitis C virus infection. Journal of Hepatology 2014;61 (1 Suppl):S45-57.

\section{Gowing 2011}

Gowing L, Farrell MF, Bornemann R, Sullivan LE, Ali $\mathrm{R}$. Oral substitution treatment of injecting opioid users for prevention of HIV infection. Cochrane Database of Systematic Reviews 2011, Issue 8. [DOI: 10.1002/ 14651858.CD004145.pub4]

\section{GRADE 2004}

The GRADE working group. Grading quality of evidence and strength of recommendations. BMJ 2004;328:1490-4.

\section{Guyatt 2008}

Guyatt GH, Oxman AD, Vist GE, Kunz R, Falck-Ytter Y, Alonso-Coello P, et al. GRADE: an emerging consensus on rating quality of evidence and strength of recommendations. BMJ 2008;336(7650):924-6.

\section{Guyatt 2011}

Guyatt G, Oxman AD, Akl EA, Kunz R, Vist G, Brozek $\mathrm{J}$, et al. GRADE guidelines 1. Introduction-GRADE evidence profiles and summary of findings tables. Journal of Clinical Epidemiology 2011;64:383-94.

\section{Hagan 2011}

Hagan H, Pouget ER, Des Jarlais DC. A systematic review and meta-analysis of interventions to prevent hepatitis C virus infection in people who inject drugs. Journal of Infectious Diseases 2011;204(1):74-83.

\section{Higgins 2011}

Higgins JPT, Green S. Cochrane Handbook for Systematic Reviews of Interventions Version 5.1.0 [updated March 2011]. The Cochrane Collaboration. Available from www.cochrane-handbook.org, 2011.

\section{Jones 2011}

Jones L, Pickering L, Sumnall H, McVeigh J, Bellis MA. A Review of the Effectiveness and Cost-effectiveness of Needle and Syringe Programmes for Injecting Drug Users. Liverpool: Centre for Public Health, Liverpool John Moores University, 2011.

\section{Kaplan 1992}

Kaplan EH, Heimer R. A model-based estimate of HIV infectivity via needle sharing. Journal of Acquired Immune Deficiency Syndromes 1992;5(11):1116-8.

\section{MacArthur 2012}

MacArthur GJ, Minozzi S, Martin N, Vickerman P, Deren $S$, Bruneau J, et al. Opiate substitution treatment and HIV transmission in people who inject drugs: systematic review and meta-analysis. BMJ 2012;345:e5945.

\section{Mathers 2012}

Mathers BM, Degenhardt L, Ali H, Wiessing L, Hickman M, Mattick RP, et al. HIV prevention, treatment, and care services for people who inject drugs: a systematic review of global, regional, and national coverage. Lancet 2010;375 (9719):1014-28.

Mohd Hanafiah 2013

Mohd Hanafiah K, Groeger J, Flaxman AD, Wiersma ST. Global epidemiology of hepatitis $\mathrm{C}$ virus infection: new estimates of age-specific antibody to HCV seroprevalence. Hepatology 2013;57(4):1333-42.

Palmateer 2010

Palmateer N, Kimber J, Hickman M, Hutchinson S, Rhodes T, Goldberg D. Evidence for the effectiveness of sterile injecting equipment provision in preventing hepatitis $\mathrm{C}$ and human immunodeficiency virus transmission among injecting drug users: a review of reviews. Addiction 2010; 105(5):844-59.

\section{Perz 2006}

Perz JF, Armstrong GL, Farrington LA, Hutin YJ, Bell BP. The contributions of hepatitis $B$ virus and hepatitis $C$ virus infections to cirrhosis and primary liver cancer worldwide. Journal of Hepatology 2006;45(4):529-38.

\section{Platt 2015}

Platt L, Easterbrook P, Gower E, McDonald B, Sabin K, McGowan C, et al. Epidemiology of HIV and HCV coinfection: a systematic review. Lancet Infectious Diseases under review 2015.

\section{Pouget 2012}

Pouget ER, Hagan H, Des Jarlais DC. Meta-analysis of hepatitis $\mathrm{C}$ seroconversion in relation to shared syringes and drug preparation equipment. Addiction 2012;107(6): 1057-65.

Schünemann 2006 Schünemann HJ, Jaeschke R, Cook D, Bria W, El-Solh A, Ernst A, et al. An official ATS statement: grading the quality of evidence and strength of recommendations in ATS guidelines and recommendations. American Journal of Respiratory and Critical Care Medicine 2006;174:605-14.

\section{Sterne 2013}

Sterne J, Higgins J, Reeves B. Extending the risk of bias tool to allow for assessment of non-randomised studies, clust-randomised trials and cross-over trials: a Cochrane methods innovation fund project (Workshop), 21st Cochrane Colloquium Abstract Book. The Cochrane Collaboration, 2013.

Effectiveness of needle/syringe programmes and opiate substitution therapy in preventing HCV transmission among people who inject 
Strathdee 2010

Strathdee SA, Hallett TB, Bobrova N, Rhodes T, Booth R, Abdool R, et al. HIV and risk environment for injecting drug users: the past, present, and future. Lancet 2010;376 (9737):268-84.

Tilson 2007

Tilson H, Aramrattana A, Bozzette SA. Preventing HIV Infection among Injecting Drug Users in High-risk Countries: An Assessment of the Evidence. Washington, DC: Institute of Medicine, 2007.

\section{Turner 2011}

Turner KM, Hutchinson S, Vickerman P, Hope V, Craine $\mathrm{N}$, Palmateer N, et al. The impact of needle and syringe provision and opiate substitution therapy on the incidence of hepatitis $\mathrm{C}$ virus in injecting drug users: pooling of UK evidence. Addiction 2011;106(11):1978-88.
UNAIDS 2014

UNAIDS. Global Statistics. Fact Sheet. UNAIDS, 2014.

Van Den Berg 2007

Van Den Berg C, Smit C, Van Brussel G, Coutinho R, Prins $M$. Full participation in harm reduction programmes is associated with decreased risk for human immunodeficiency virus and hepatitis $\mathrm{C}$ virus: evidence from the Amsterdam Cohort Studies among drug users. Addiction 2007;102(9): 1452-62.

\section{Wodak 2004}

Wodak A, Cooney A. Effectiveness of sterile needle and syringe programming in reducing HIV/AIDS among injecting drug users. Evidence for Action Technical Papers. Geneva: World Health Organisation, 2004.

* Indicates the major publication for the study

\section{A P P E N D I C E S}

\section{Appendix I. MEDLINE search strategy I}

1. Needle-Exchange Programs/

2. Community pharmacy services/

3. ((needle* or syringe* or inject $\left.^{*}\right)$ adj3 exchange).ab,ti.

4. Harm Reduction/

5. (harm adj reduc*).ab,ti.

6. ((needle* or syringe* or inject*) adj3 (suppl* or access* ${ }^{*}$ or provision or provid* or distribut* ${ }^{*}$ or dispens* or pack $\left.^{*}\right)$ ).ab,ti.

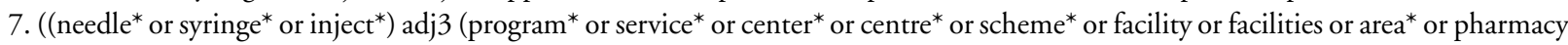
or pharmacies or unit or units or room*)).ab,ti.

8. ((needle* or syringe* or inject* or slot or dispensing or vending) adj3 (machine* or (peer adj distrib*))).ab,ti.

9. or/1-8

10. Substance Abuse, Intravenous/

11. (substance $\$$ or drug\$).ab,ti.

12. (abuse $\$$ or depend $\$$ or use $\$$ or misus $\$$ or addict $\$$ ).ab,ti.

13. (inject\$ or intravenous).ab,ti.

14. 10 or (11 and 12) or (11 and 13)

15. opiate substitution treatment/

16. methadone/

17. buprenorphine/

18. (((substitut* or maint*) adj2 (treatment or therapy)) or methadone or buprenorphine or subutex or suboxone).ab,ti.

19. or/ $15-18$

20. $\exp$ Hepatitis C/

21. (hepatitis-c or or hep c or hcv).ab,ti.

22. 20 or 21

23. (9 or 19$)$ and 14 and 22

Effectiveness of needle/syringe programmes and opiate substitution therapy in preventing HCV transmission among people who inject 


\section{Appendix 2. MEDLINE search strategy 2}

1. Substance Abuse, Intravenous/

2. (substance $\$$ or drug $\$$ ).ab,ti.

3. (abuse $\$$ or depend $\$$ or use $\$$ or misus $\$$ or addict $\$$ ).ab,ti.

4. (inject\$ or intravenous).ab,ti.

5. 1 or (2 and 3$)$ or (2 and 4$)$

6. exp Hepatitis C/

7. (hepatitis-c or hcv).ab,ti.

8. (HCV adj2 seroconvers\$).ti,ab.

9. (HCV adj2 transmission).ti,ab.

10. or/6-9

11. exp Cohort Studies/

12. exp Longitudinal Studies/

13. (prospective or longitudinal or cohort).ti,ab.

14. or/11-13

15. 5 and 10 and 14

16. Animals/

17. 15 not 16

\section{Appendix 3. Criteria for risk of bias assessment for RCTs}

\begin{tabular}{|c|c|c|}
\hline Item & Judgment & Description \\
\hline \multirow[t]{3}{*}{$\begin{array}{l}\text { 1. Random sequence generation (selection } \\
\text { bias) }\end{array}$} & Low risk & $\begin{array}{l}\text { The investigators describe a random component in the sequence gener- } \\
\text { ation process such as: random number table; computer random num- } \\
\text { ber generator; coin tossing; shuffling cards or envelopes; throwing dice; } \\
\text { drawing of lots; minimisation }\end{array}$ \\
\hline & High risk & $\begin{array}{l}\text { The investigators describe a non-random component in the sequence } \\
\text { generation process such as: odd or even date of birth; date (or day) of } \\
\text { admission; hospital or clinic record number; alternation; judgement of } \\
\text { the clinician; results of a laboratory test or a series of tests; availability of } \\
\text { the intervention }\end{array}$ \\
\hline & Unclear risk & $\begin{array}{l}\text { Insufficient information about the sequence generation process to permit } \\
\text { judgement of low or high risk }\end{array}$ \\
\hline \multirow[t]{2}{*}{ 2. Allocation concealment (selection bias) } & Low risk & $\begin{array}{l}\text { Investigators enrolling participants could not foresee assignment because } \\
\text { one of the following, or an equivalent method, was used to conceal alloca- } \\
\text { tion: central allocation (including telephone, web-based, and pharmacy- } \\
\text { controlled, randomisation); sequentially-numbered drug containers of } \\
\text { identical appearance; sequentially-numbered, opaque, sealed envelopes }\end{array}$ \\
\hline & High risk & $\begin{array}{l}\text { Investigators enrolling participants could possibly foresee assignments } \\
\text { because one of the following method was used: open random allocation } \\
\text { schedule (e.g. a list of random numbers); assignment envelopes without } \\
\text { appropriate safeguards (e.g. if envelopes were unsealed or nonopaque or } \\
\text { not sequentially numbered); alternation or rotation; date of birth; case }\end{array}$ \\
\hline
\end{tabular}

Effectiveness of needle/syringe programmes and opiate substitution therapy in preventing HCV transmission among people who inject drugs (Protocol)

Copyright $(2016$ The Cochrane Collaboration. Published by John Wiley \& Sons, Ltd. 
record number; any other explicitly unconcealed procedure

Unclear risk Insufficient information to permit judgement of low or high risk. This is usually the case if the method of concealment is not described or not described in sufficient detail to allow a definite judgement

3. Blinding of participants and providers Low risk (performance bias)

Objective outcomes
No blinding or incomplete blinding, but the review authors judge that the outcome is not likely to be influenced by lack of blinding;

Blinding of participants and key study personnel ensured, and unlikely that the blinding could have been broken

High risk No blinding or incomplete blinding, and the outcome is likely to be influenced by lack of blinding;

Blinding of key study participants and personnel attempted, but likely that the blinding could have been broken, and the outcome is likely to be influenced by lack of blinding

Unclear risk Insufficient information to permit judgement of low or high risk

4. Blinding of participants and providers Low risk Blinding of participants and providers ensured and unlikely that the blinding could have been broken

Subjective outcomes

High risk No blinding or incomplete blinding, and the outcome is likely to be influenced by lack of blinding;

Blinding of key study participants and personnel attempted, but likely that the blinding could have been broken, and the outcome is likely to be influenced by lack of blinding

Unclear risk Insufficient information to permit judgement of low or high risk

5. Blinding of outcome assessor (detection Low risk bias)

Objective outcomes

No blinding of outcome assessment, but the review authors judge that the outcome measurement is not likely to be influenced by lack of blinding; Blinding of outcome assessment ensured, and unlikely that the blinding could have been broken

High risk No blinding of outcome assessment, and the outcome measurement is likely to be influenced by lack of blinding;

Blinding of outcome assessment, but likely that the blinding could have been broken, and the outcome measurement is likely to be influenced by lack of blinding

Unclear risk Insufficient information to permit judgement of low or high risk

6.Blinding of outcome assessor (detection Low risk bias)

Subjective outcomes
Blinding of outcome assessment ensured, and unlikely that the blinding could have been broken 
High risk No blinding of outcome assessment, and the outcome measurement is likely to be influenced by lack of blinding;

Blinding of outcome assessment, but likely that the blinding could have been broken, and the outcome measurement is likely to be influenced by lack of blinding

Unclear risk Insufficient information to permit judgement of low or high risk

7. Incomplete outcome data (attrition bias) For all outcomes except retention in treatment or drop out

Low risk ment or drop out
No missing outcome data;

Reasons for missing outcome data unlikely to be related to true outcome (for survival data, censoring unlikely to be introducing bias);

Missing outcome data balanced in numbers across intervention groups, with similar reasons for missing data across groups;

For dichotomous outcome data, the proportion of missing outcomes compared with observed event risk not enough to have a clinically-relevant impact on the intervention effect estimate;

For continuous outcome data, plausible effect size (difference in means or standardised difference in means) among missing outcomes not enough to have a clinically-relevant impact on observed effect size;

Missing data have been imputed using appropriate methods;

All randomised patients are reported/analysed in the group they were allocated to by randomisation irrespective of non-compliance and cointerventions (intention to treat)

High risk Reason for missing outcome data likely to be related to true outcome, with either imbalance in numbers or reasons for missing data across intervention groups;

For dichotomous outcome data, the proportion of missing outcomes compared with observed event risk enough to induce clinically relevant bias in intervention effect estimate;

For continuous outcome data, plausible effect size (difference in means or standardised difference in means) among missing outcomes enough to induce clinically relevant bias in observed effect size;

'As-treated' analysis done with substantial departure of the intervention received from that assigned at randomisation

Unclear risk Insufficient information to permit judgement of low or high risk (e.g. number randomised not stated, no reasons for missing data provided; number of drop out not reported for each group)

8 Selective reporting (reporting bias) Low risk
The study protocol is available and all of the study's pre-specified (primary and secondary) outcomes that are of interest in the review have been reported in the pre-specified way;

The study protocol is not available but it is clear that the published reports include all expected outcomes, including those that were pre-specified (convincing text of this nature may be uncommon) 
High risk Not all of the study's pre-specified primary outcomes have been reported; One or more primary outcomes is reported using measurements, analysis methods or subsets of the data (e.g. subscales) that were not pre-specified; One or more reported primary outcomes were not pre-specified (unless clear justification for their reporting is provided, such as an unexpected adverse effect);

One or more outcomes of interest in the review are reported incompletely so that they cannot be entered in a meta-analysis;

The study report fails to include results for a key outcome that would be expected to have been reported for such a study

Unclear risk Insufficient information to permit judgement of low or high risk

\section{WHAT'S NEW}

\begin{tabular}{l|l|l}
\hline Date & Event & Description \\
\hline 20 January 2016 & Amended & External source of support added \\
\hline
\end{tabular}

\section{CONTRIBUTIONSOFAUTHORS}

All authors contributed to this protocol.

\section{DECLARATIONSOF INTEREST}

Lucy Platt: None known

Jennifer Reed: None known

Silvia Minozzi: None known

Peter Vickerman: None known

Holly Hagan: None known

Clare French: None known

Ashly Jordan: None known

Louisa Degenhardt: I have received untied educational grants from Reckitt Benckiser for the post-marketing surveillance of buprenorphine-naloxone tablets and soluble film (2006-2013), the development of an opioid-related behavior scale (2010), and from Mundipharma for the conduct of post-marketing surveillance studies following the introduction of a new formulation of oxycodone in Australia. All such studies? design, conduct and interpretation of findings are the work of the investigators; the funders had no role in these. They had no knowledge of this work.

Vivian Hope: None known

Effectiveness of needle/syringe programmes and opiate substitution therapy in preventing HCV transmission among people who inject 
Sharon Hutchinson: Outside the submitted work, received honoraria from pharma (Abbvie and Gilead) for speaking at conferences/ meetings on the epidemiology and treatment of HCV infection.

Lisa Maher: None known

Norah Palmateer: None known

Avril Taylor: The Scottish Government funded the "Needle Exchange Surveillance Initiative". Some of the data from this is used in the paper under consideration

Matthew Hickman: None known

\section{SOURCES OF SUPPORT}

\section{Internal sources}

- No sources of support supplied

\section{External sources}

- European Commission Drug Prevention and Information Programme (DIPP) Grant "Treatment as Prevention in Europe: Model Projections of Impact And Strengthening Evidence Base On Intervention Coverage and Effect and HCV Morbidity". [JUST/ 2013/DPIP/AG/4812], Other.

- National Institutes of Health/ National Institute on Drug Abuse (NIDA), USA.

Holly Hagan, Ashly Jordan and Jennifer Reed are supported by NIH-NIDA grant [1 R01 DA034637]

- National Institute of Health Research (NIHR), UK.

Clare French was funded by the NIHR Health Protection Research Unit in Evaluation of Interventions at University of Bristol (grant number: HPRU-2012-10026). The views expressed are those of the author(s) and not necessarily those of the NHS, the NIHR, the Department of Health or Public Health England.

- Lisa Maher is supported by an Australian National Health and Medical Research Council Senior Research Fellowship, Australia. 